\title{
Diagnostic biomarkers and potential drug targets for coronary artery disease as revealed by systematic analysis of IncRNA characteristics
}

\author{
Ziqi Chen ${ }^{1}$, Dawang Zhou ${ }^{2}$, Xiaocong Zhang ${ }^{1,3}$, Qian $W_{u^{4}}$, Guifu $W^{1,5,6}$ \\ ${ }^{1}$ Department of Cardiology, The Eighth Affiliated Hospital of Sun Yat-sen University, Shenzhen, China; ${ }^{2}$ Department of Emergency, The \\ Seventh Affiliated Hospital of Sun Yat-sen University, Shenzhen, China; ${ }^{3}$ Department of Cardiology, Sun Yat-Sen Memorial Hospital of Sun Yat- \\ sen University, Guangzhou, China; ${ }^{4}$ Department of Gerontology, Guangzhou First People's Hospital of South China University of Technology, \\ Guangzhou, China; ${ }^{5}$ Guangdong Innovative Engineering and Technology Research Center for Assisted Circulation, Shenzhen, China; ${ }^{6} \mathrm{NHC}$ Key \\ Laboratory of Assisted Circulation (Sun Yat-sen University), Guangzhou, China \\ Contributions: (I) Conception and design: G Wu, Z Chen; (II) Administrative support: Q Wu; (III) Provision of study materials or patients: D Zhou; (IV) \\ Collection and assembly of data: Z Chen, X Zhang; (V) Data analysis and interpretation: G Wu; (VI) Manuscript writing: All authors; (VII) Final \\ approval of manuscript: All authors. \\ Correspondence to: Guifu Wu. Department of Cardiology, The Eighth Affiliated Hospital of Sun Yat-Sen University, Shenzhen 518000, China. \\ Email: wuguifu@mail.sysu.edu.cn.
}

Background: The expression profile of lncRNAs in coronary artery disease (CAD) patients has not yet been fully explored. Therefore, the current study aimed to investigate lncRNA-based prognostic biomarkers for CAD.

Methods: The expression profiles of lncRNA and messenger RNA (mRNA) were downloaded from the Gene Expression Omnibus (GEO) database. Differentially expressed lncRNA (DElncRNAs) and DEmRNAs were identified from CAD and normal samples, and weighted gene co-expression network analysis (WGCNA) was conducted. Gene Ontology (GO) and Kyoto Encyclopedia of Genes and Genomes (KEGG) pathway enrichment analyses were performed to investigate the principal functions of significantly dysregulated genes. The potential drugs of new CAD-specific genes were identified by network distance method. Receiver operating characteristic (ROC) was used to verify the classification performance of genes.

Results: A total of 512 differentially expressed genes (DEGs) and 308 DElncRNAs were identified from GSE113079 dataset to classify CAD samples. Through WGCNA co-expression analysis, 24 co-expression modules were obtained. A total of 187 DElncRNAs and 253 DEGs were determined from 7 modules correlated with CAD. Functional enrichment analysis showed that these DEGs were mainly related to inflammatory and immune-related pathways. Furthermore, 36 regulatory pairs of significantly shared micro RNAs (miRNAs) were identified as dysregulated lncRNA-mRNA (LRM-CAD), which contained 11 lncRNAs and 33 genes. Compared with a single lncRNA or gene, LRM-CAD showed stronger classification performance [average area under the curve $(\mathrm{AUC})=0.958]$. We screened 3 potential therapeutic drugs, DB09105, DB12371, and DB12612, a by binding drug-target gene interaction network. Molecular docking verified that the S1PR1 gene bound relatively closely to DB12371 and DB12612. The ROC analysis on external data sets showed that S1PR1, AC012640.4, and S1PR1-AC012640.4 could effectively distinguish CAD samples from control samples.

Conclusions: We provided a transcriptome overview of abnormally expressed lncRNAs in CAD patients and identified novel biomarkers for diagnosing CAD.

Keywords: Long noncoding RNAs (lncRNAs); coronary artery disease (CAD); expression profile; S1PR1; Gene Expression Omnibus (GEO)

Submitted Jun 02, 2021. Accepted for publication Aug 02, 2021.

doi: $10.21037 / \mathrm{atm}-21-3276$

View this article at: https://dx.doi.org/10.21037/atm-21-3276 


\section{Introduction}

Coronary artery disease (CAD) is a complex, multifactorial disease that remains 1 of the most common causes of death (1). Many environmental and genetic factors, including age, smoking behavior, hypertension, dyslipidemia, obesity, diabetes, and family history, contribute to CAD (2-4). However, the number of biomarkers used clinically to predict the incidence of CAD is limited at this stage (5). Currently, coronary angiography is a standard for diagnosing coronary artery diseases; however, these tests sometimes cause significant changes and severe complications, such as vagal reflex, vasospasm, puncture site-related complications and kidney dysfunction. The latency and non-specificity of traditional screening programs also limit their use in clinical practice.

Recent studies have shown that IncRNAs are functional RNA molecules with a length of more than 200 nucleotides, which are generally not translated into proteins, and their expression patterns are either high or low, but they can regulate the expression and function of protein-coding genes through different mechanisms, such as ceRNA model. Many lncRNAs are involved in cardiovascular pathophysiology and have been inferred as potential therapeutic targets. Long noncoding RNAs (lncRNAs) are transcriptional products containing 200 nucleotides and are involved in the etiology of many human diseases through epigenetic, transcriptional, and post-transcriptional regulation $(6,7)$. Interestingly, lncRNAs also appear to participate in the development of cardiovascular disease, including heart failure, cardiac hypertrophy, cardiomyopathy, and myocardial infarction (8). The IncRNAs alter the expression of related proteins through RNA interference, gene silencing, chromatin remodeling, DNA methylation, and other pathways (9). In addition, the function of lncRNAs is usually mediated through regulating microRNAs (miRNAs), which regulate gene expression posttranscriptionally by binding to the 3 ' untranslated region (UTR) of messenger RNA (mRNA) (10).

The gene S1PR1 is a member of the G protein-coupled receptors group, including S1PR1-5, which are widely expressed in endothelial cells and vascular smooth muscle cells, and is involved in the regulation of various vascular physiological activities. Especially in the cardiovascular system, the proportion of S1PR1 is the highest. The lumen S1PR1 can activate Pertussis toxin-sensitive G proteins, activate Rac1 signaling pathway, and eventually lead to endothelial cytoskeleton rearrangement, thereby promoting endothelial cell migration and proliferation (11). The anti- atherosclerosis effect of high density lipoprotein (HDL) is well known, and HDL is the main carrier of S1P in plasma (12). Binding to HDL, S1P promotes the formation of S1P1- $\beta$-Arrestin 2 complex on cell surface and inhibits TNF- $\alpha$ production through S1PR1 signaling. Thus inhibiting the pro-inflammatory response of endothelial cells and vascular smooth muscle cells. In addition, an increasing number of studies have shown that S1PR1 couples to Gi and activates downstream signaling pathways, including the PI3K/AKT, PI3K/Rac, Ras/ERK, NF- $\mathrm{KB}$, and PLC signaling pathways $(13,14)$.

The aim of this study was to investigate the potential function of lncRNA, mRNA expressions in CAD using RNA expression profiles of CAD patients. We systematically analyzed lncRNA and mRNA expression profiles between $\mathrm{CAD}$ and healthy patients. Furthermore, a novel algorithm was proposed for the identification of dysregulated IncRNAmRNA (LRM-CAD) during CAD progression, so as to discover biomarkers as potentially effective therapeutic agents for $\mathrm{CAD}$ diagnosis and prognosis. We present the following article in accordance with the TRIPOD reporting checklist (available at https://dx.doi.org/10.21037/atm-21-3276).

\section{Methods}

This study consisted of data collection, variance analysis, co-expression module identification, enrichment analysis, feature selection, and classifier construction and validation. The workflow is shown in Figure 1.

\section{$R N A$ expression spectrum}

The lncRNA expression profiles of CAD were downloaded from the Gene Expression Omnibus (GEO) database (http://www.ncbi.nlm.nih.gov/geo/) under the number GSE113079 (15) on the Agilent-067406 Human CBC lncRNA + mRNA microarray V4.0 (Agilent Tech., Santa Clara, CA, USA), which was a dataset of 141 samples with whole blood samples from 93 CAD patients and 48 normal controls. In addition, we also downloaded the gene expression profile data sets GSE20681 (16) and GSE64566 (17) between CAD and control of 2 other platforms. The GSE20681 dataset was derived from Agilent-014850 Whole Human Genome Microarray 4x44K G4112F (Agilent Tech. Feature Number version) platform and contained 99 CAD samples and 99 controls. The GSE64566 dataset was derived from Illumina HumanHT-12 V3.0 Expression BeadChip platform 


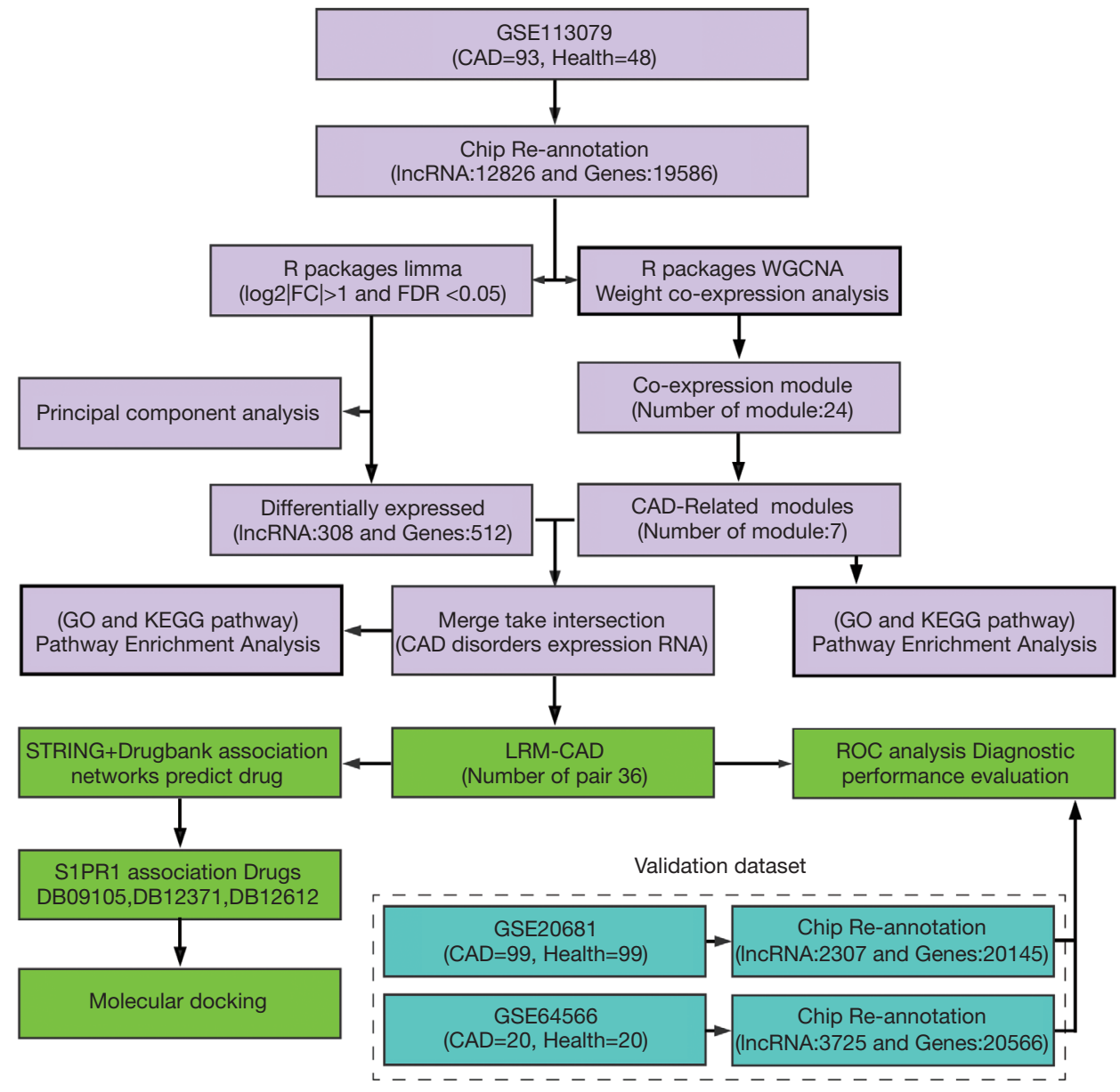

Figure 1 Workflow. CAD, coronary artery disease; WGCNA, weighted gene co-expression network analysis; GO, Gene Ontology; KEGG, Kyoto Encyclopedia of Genes and Genomes; ROC, receiver operating characteristic.

(Illumina, San Diego, CA, USA) and contained 26 CAD samples and 20 control samples.

Probe sequences from the 3 datasets were matched to the genome by microarray re-annotation (version GRCh38. p13) to obtain transcript IDs for probe mapping, and each transcript cluster was assigned with an Ensembl gene ID. For transcript clusters with Ensembl gene IDs, we retained the annotation types "lincRNA", "sense_intronic", "sense_ overlapping", "antisense", "processed_transcript", "3prime_ overlapping_ncRNA" clusters were considered as lncRNAs, and clusters with annotation type "protein_coding" were considered to be coding genes (18). Finally, for mRNA, lncRNA expression profiles, probes were mapped to genes/ lncRNAs, and when multiple probes were mapped to the same gene/lncRNA, the median expression value was taken as the expression value of the gene/lncRNA.

\section{Differential expression analysis and weighted co-expression network}

The R software package Limma (19) was used to screen differentially expressed genes (DEGs) and lncRNAs between $\mathrm{CAD}$ and control samples. Firstly, the expression profile of the GSE113079 dataset was analyzed. To obtain biologically different genes, false discovery rate (FDR) $<0.05$ and double difference were the thresholds to identify DEGs and lncRNAs (DElncRNAs). At the same time, based on DEG and DElncRNAs expression profiles, the $\mathrm{R}$ software package ggfortify was used for principal component analysis (PCA) to determine the classification performance of DEG/DElncRNAs for CAD. In addition, for the purpose of better identifying diseaserelated genes and lncRNAs, we combined the expression profiles of lncRNAs and genes to build a weighted co- 
expression module. Specifically, RNA expression data profile of genes/lncRNAs was used to evaluate whether the samples and genes/lncRNAs were qualified subjects. Then, we used the weighted gene co-expression network analysis (WGCNA) (20) package in R to construct a scalefree co-expression network for the genes/lncRNAs. The Pearson's correlation matrices and average linkage method were both performed for all pair-wise analyses. Then, a weighted adjacency matrix was constructed using power function $\mathrm{A}_{m n}=\left|\mathrm{C}_{m n}\right|^{\beta}\left(\mathrm{C}_{m n}=\right.$ Pearson's correlation between gene/lncRNA $\mathrm{m}$ and gene/lncRNA $\mathrm{n} ; \mathrm{A}_{m n}=$ adjacency between gene/lncRNA $\mathrm{m}$ and gene/lncRNA $\mathrm{n}$ ). The $\beta$ was a soft-thresholding parameter emphasizing strong correlations between gene/lncRNAs and to penalize weak correlations. After choosing the power of $\beta$, the adjacency was transformed into a topological overlap matrix (TOM) to measure the network connectivity of a gene/lncRNA that was defined as the sum of its adjacency with all other gene/lncRNA for network gene/lncRNA ratio. Then the corresponding dissimilarity (1-TOM) was calculated. To classify gene/lncRNA with similar expression profiles into gene/lncRNA modules, average linkage hierarchical clustering was conducted for the gene/lncRNAs dendrogram, according to the TOM-based dissimilarity measure with a minimum size (gene/lncRNA group) set at 30 . The module was further analyzed by calculating the dissimilarity of module eigengene (ME)/lncRNAs, and a cut line was chosen for module dendrogram to merge some modules.

\section{Identification of disease-related co-expression modules}

We defined the module associated with the occurrence of CAD as Co-DGL Module, and the genes and lncRNAs in the Co-DGL Module were differentially co-expressed DEG/L. We used 3 methods to determine the correlation between disease and modules. We first calculated the Spearman correlation coefficient of each gene/lncRNA expression in each co-expression module of CAD, and selected the mean value of the correlation coefficient greater than the overall mean value of all modules. Furthermore, the correlation between CAD and each MEs was calculated, and the significantly correlated modules were selected. Finally, the distribution of each MEs in the CAD and control groups was analyzed to determine the modules with significant differences. The intersection sets were taken based on the above 3 methods to identify the key modules for the disease.

\section{Functional enrichment analyses and gene set enrichment analysis}

Gene Ontology (GO) and Kyoto Encyclopedia of Genes and Genomes (KEGG) pathway enrichment analysis was performed using the $\mathrm{R}$ packages clusterProfiler (21) on genes significantly associated with CAD-related modules. Then, over-represented GO terms in 3 categories (biological processes, molecular function and cellular component) and KEGG pathway were identified. For both analyses, a q-value of $<0.05$ was considered to denote statistical significance.

The R software package GSVA (22) was used for enrichment analysis of single sample gene sets, and gene expression profiles were used to evaluate the enrichment scores of each sample in different KEGG pathways.

\section{Regulatory interactions between miRNA-mRNA and miRNA-lncRNA}

The miRNA-mRNA regulatory relationships were collected from miRanda (23), miRTarBase (24), TargetScan (25) and starBase (26) databases, and 416,312 non-redundant miRNA-mRNA interactions were obtained. The miRNAlncRNA interactions were retrieved from starBase (26) and miRcode (27) databases, and 295,601 non-redundant miRNA-lncRNA relationships were retained.

\section{LRM-CAD}

We speculated that there were LRM-CAD, and dysfunction of which would possibly lead to the occurrence and development of CAD. Based on the competing endogenous RNA (ceRNA) hypothesis $(28,29)$, a candidate LRM-CAD is defined if it satisfies all the following conditions: (I) the miRNA shared by mRNA and lncRNA is significantly enriched (determined by the hypergeometric test, $\mathrm{P}<0.05$ ); (II) mRNA-lncRNA is in the same disease-related coexpression module. (III) Both mRNA and lncRNA are differentially expressed in CAD samples.

In addition, the $\mathrm{R}$ software package GSVA (22) was used for single-sample gene-set enrichment analysis. Gene expression profiles were used to evaluate the gene-set and lncRNAs enrichment scores of each sample in LRM-CAD, and to compare their differences in tumor and control samples and their relationship with the KEGG pathway. Next, the gene sets and lncRNAs in LRM-CAD were mapped to the genome and visualized using the $\mathrm{R}$ package 
OmicCircos (30).

\section{Identification of potential drugs targeting LRM-CAD}

To observe potential drugs targeting LRM-CAD, 5,490 druggene interaction data were obtained from Drugbank (31) using the method previously described by Peng et al. (32). These proteins and genes in LRM-CAD were mapped to the Search Tool for the Retrieval of Interacting Genes/Proteins (STRING) database (33) to construct drug-protein and protein-protein interaction network (DPPI) and used to define the degree of node of LRM-CAD-related gene set in PPI, T, and drug target gene set. Distance d (S,T) qA the shortest path between $S$ node and $T$ node (where $s \in S$, is LRM-CAD related gene; $t \in T$, is a drug target gene), and the calculation method was as follows:

$$
d(S, T)=\frac{1}{|T|} \sum_{t \in T} \min _{s \in S}(d(s, t)+\omega)
$$

Where $\omega$ is the weight of the target gene, if the target gene is a gene in the LRM-CAD related gene set, the calculation method is $\omega=-\ln (\mathrm{D}+1)$, otherwise $\omega=0$.

In addition, a simulated reference distance distribution corresponding to the drug was generated. In short, a group of protein nodes were randomly selected from the network as the simulated drug target, with the same number of nodes as the target size (denoted by $\mathrm{R}$ ). Then, the distance $\mathrm{d}$ ( $\mathrm{S}$, $\mathrm{R})$ between the simulated drug targets (representing the simulated drug) and LRM-CAD was calculated. After 10,000 random repeats, the simulated reference distribution was generated. At the same time, the mean and standard deviation $(\mathrm{SD})$ of the $\mu \mathrm{D}(\mathrm{S}, \mathrm{R})$ and $\sigma(\mathrm{S}, \mathrm{R})$ reference distribution and the actual observation distance were used to convert to the standardized score, that was, the proximity degree z:

$$
z(S, T)=\frac{d(S, T)-\mu d(S, R)}{\sigma d(S, R)}
$$

The proximity distribution in the actual network and the random network were evaluated, and the significance $\mathrm{P}$ value of the proximity of each drug was calculated. Drugs with a global significance FDR $<0.01$ were regarded as the final potential drug candidates targeting LRM-CAD.

\section{Evaluation of diagnostic performance and predictive ability of LRM-CAD}

For the genes and lncRNAs in LRM-CAD of the training set, the R software package plotROC was used to analyze the expression of each lncRNA and gene, and the Receiver operating characteristic (ROC) analysis of CAD and control sample classification was visualized. For each LRM$\mathrm{CAD}$, the expression profiles of corresponding genes and LRM-CAD were extracted, and linear discriminant analysis was used to establish a linear model. plotROC was used to analyze each LRM-CAD for ROC analysis and to visualize CAD and control sample classification. The predictive ability of each IncRNA, gene, and LRM-CAD was evaluated using the area under the ROC (AUC). In addition, GSE20681 and GSE124272 served as external validation sets.

\section{Data availability}

The discovered Gene Expression data were downloaded from the GEO, numbers GSE64566, GSE20681, and a standardized data set were downloaded. Genome annotation files were download from GENCODE (https://www.gencodegenes.org/human/). The regulatory relationships between miRNA-mRNA were downloaded from miRWalk (http://mirwalk.umm.uni-heidelberg.de/). Regulatory interactions between miRNA-lncRNA were downloaded from starBase (http://starbase.sysu.edu.cn/) and miRcode (http://www.mircode.org/). Drug and protein interaction data were downloaded from Drugbank (https:// go.drugbank.com/).

\section{Code availability}

We host all the codes involved in the manuscript on GitHub, codes are available at https://github.com/chenzqstar/CADLncRNA.

\section{Ethical statement}

The study was conducted in accordance with the Declaration of Helsinki (as revised in 2013).

\section{Statistical analysis}

The R package pROC is used for AUC analysis, and the R package ComplexHeatmap is used for heat map drawing. All analysis, except for special instructions, uses default parameters, and data visualization is performed using ggplot2 in version 3.4.3 of $\mathrm{R}$ software. 

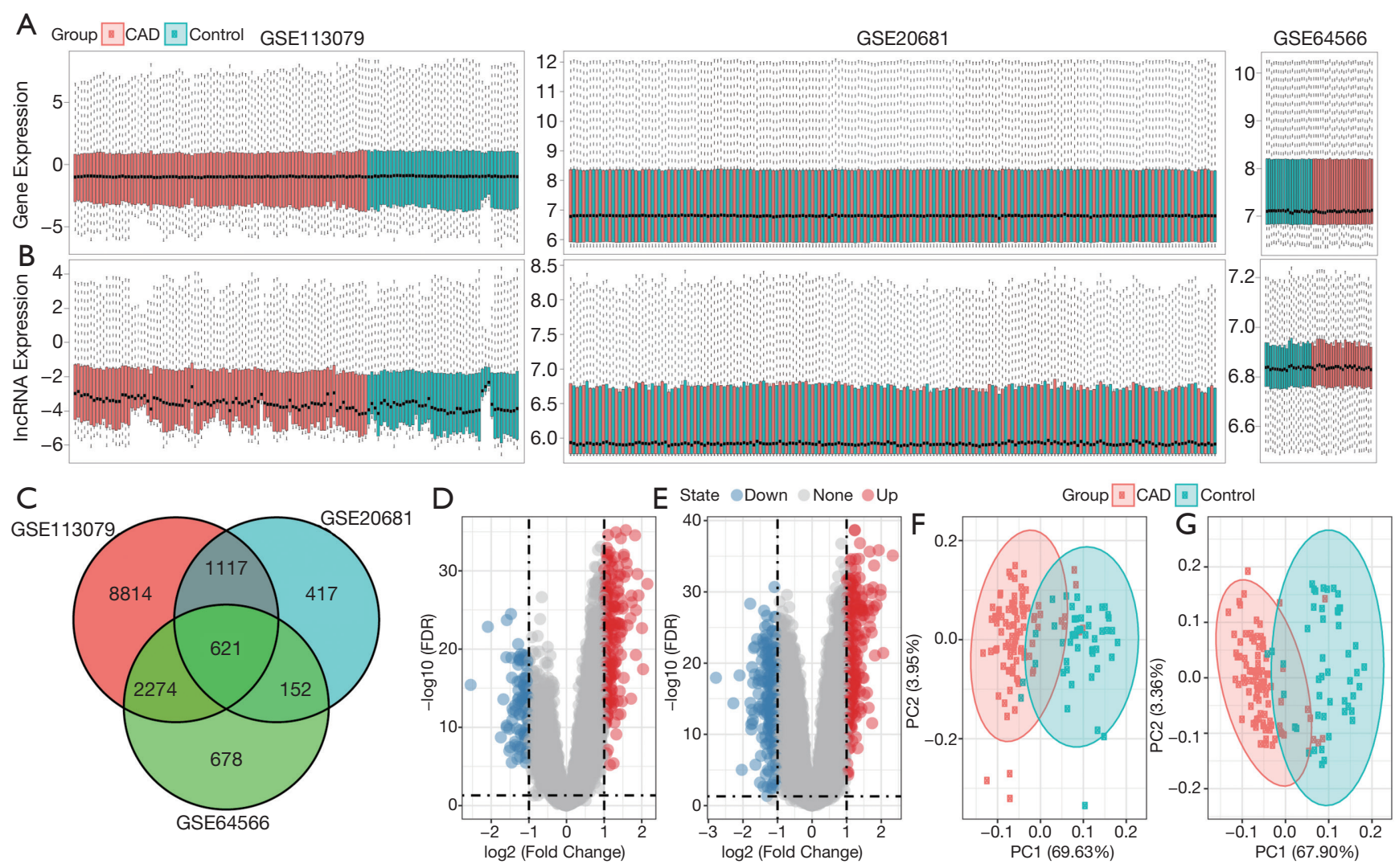

Figure 2 Data standardization and DEG analysis. (A) Expression distribution of protein-coding genes in the three datasets. (B) Expression distribution of lncRNAs in the 3 datasets. (C) Intersection Venn diagram of lncRNAs in the 3 datasets. (D) Differential expression volcano map of lncRNAs in the GSE113079 dataset. (E) Differential expression volcano map of protein-coding genes in the GSE113079 dataset. (F) PCA of differential lncRNA expression profiles in the GSE113079 dataset. (G) PCA of differential gene expression profiles in the GSE113079 dataset. DEG, differentially expressed gene; PCA, principal component analysis.

\section{Results}

\section{Identification of DEGs/differentially expressed lncRNAs (DElncRNAs) between CAD samples and bealthy controls samples}

After data standardization and chip reannotation, the expression profiles of $12,826 \operatorname{lncRNAs}$ and 19,586 genes were obtained from the GSE113079 dataset, the expression profiles of 2,307 lncRNAs and 20,045 genes were obtained from the GSE20681 dataset, and the expression profiles of 3,725 lncRNAs and 20,566 genes were obtained from the GSE64566 dataset (Figure 2A,2B). The median expression level of lncRNAs in all samples was generally lower than that of protein-coding genes. In addition, we compared the intersection of lncRNAs in the 3 datasets, and observed that the coincidence degree of the identified lncRNAs was relatively low in the datasets from different platforms
(Figure 2C). Therefore, in this study, GSE113079, which was a data set with high coincidence degree of lncRNA with other data sets, was selected as the training set, and GSE20681 and GSE124272 were the verification data sets. A total of 512 DEGs and 308 DElncRNAs were identified in the training set (Figure 2D,2E). The PCA using these differential genes and lncRNA expression profiles showed that the first and second principal components could clearly distinguish CAD samples from control samples (Figure 2F,2G), suggesting that these DEGs and DELs had certain diagnostic performance.

\section{Construction of weighted co-expression network and identification of CAD-related modules}

Gene/lncRNAs with similar expression patterns were grouped into modules by means of average linkage 
A

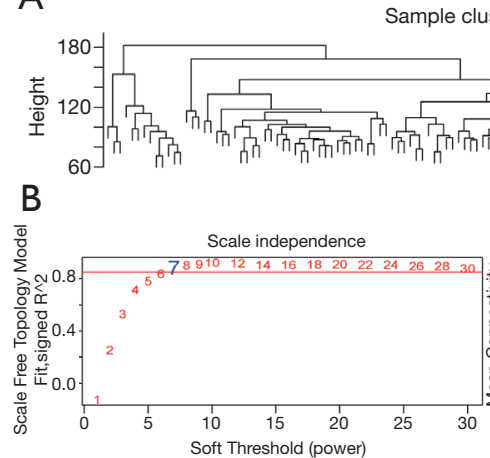

C

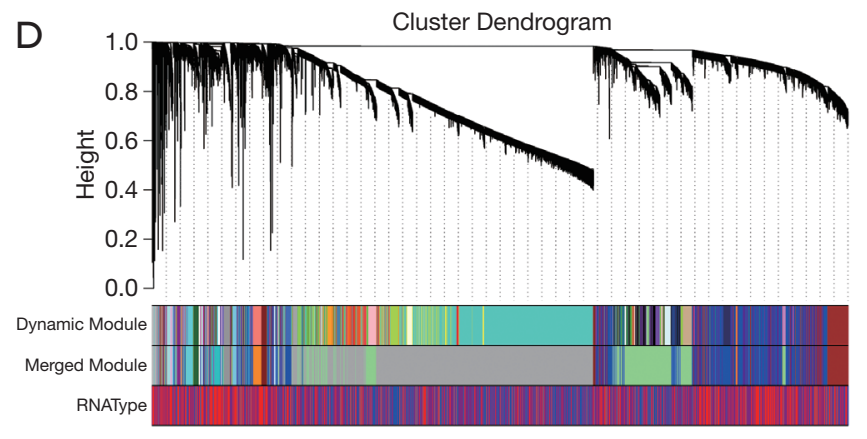

$\mathrm{E}$

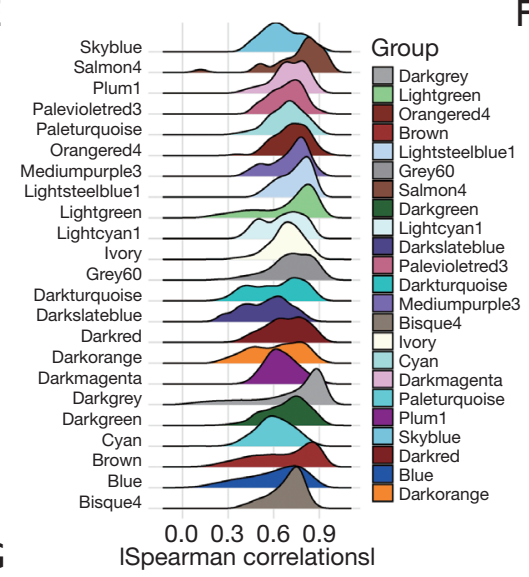

$\mathrm{F}$

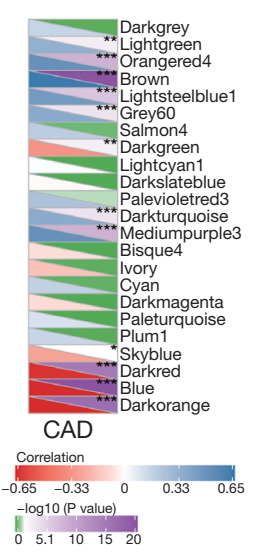

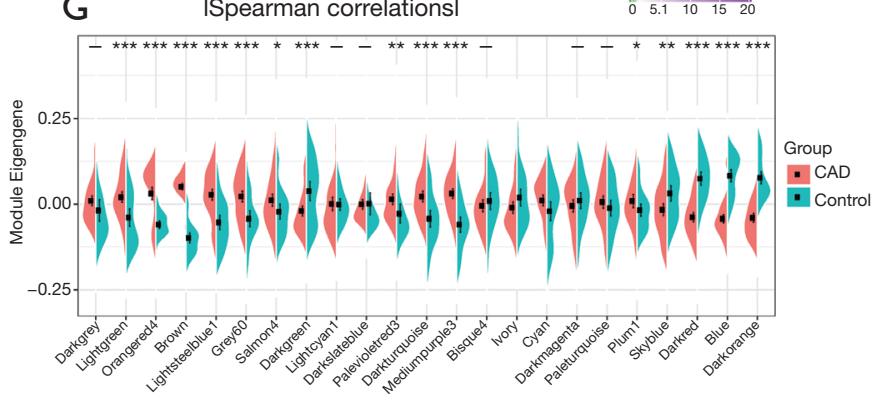

Figure 3 Construction of weighted co-expression network and identification of CAD related modules. (A) Cluster tree of each sample. (B) Analysis of the scale-free fit index for various soft-thresholding powers ( $\beta$ ). (C) Analysis of the mean connectivity for various softthresholding powers. (D) Dendrogram of all DEGs/DElncRNAs clustered based on a dissimilarity measure (1-TOM). (E) Correlation distribution of genes and eigenvectors of corresponding modules in each module; (F) correlation between each module and CAD; (G) distribution of feature vectors of each module in CAD and control samples. CAD, coronary artery disease; DEG, differentially expressed gene; DElncRNA, differentially expressed long non-coding RNA. ${ }^{*} \mathrm{P}<0.05,{ }^{* *} \mathrm{P}<0.01,{ }^{* * *} \mathrm{P}<0.001$.

hierarchical clustering using the "WGCNA" package in R. In this study, the clustering distance between each sample (Figure $3 A$ ) was firstly analyzed, and the distance was similar without outlier samples. A power of $\beta=7$ (scalefree $\mathrm{R}^{\wedge} 2=0.88$ ) was the soft threshold to ensure a scale-free network (Figure 3B,3C). Here, a total of 24 modules were identified (Figure 3D). We used 3 methods to determine the correlation between diseases and modules. The Spearman correlation coefficient between gene/lncRNA and disease occurrence in each module as well as the correlation between modules and disease occurrence were calculated (Figure $3 E$ ). We selected modules with a mean value of correlation coefficient greater than the overall mean value of all modules. Furthermore, the correlation between CAD and each module was determined to select the module with significant correlation (Figure 3F). Finally, the distribution difference of the feature vectors of each module in CAD and the control group was analyzed (Figure 3G), and the module with significant difference was selected. Based on the above 3 methods, brown, darkgreen, grey60, lightgreen, lightsteelblue1, mediumpurple3, and orangered 4 module were identified as the key modules of the disease.

\section{Functional implications of CAD-related modules}

To better understand the functional implications of the 7 disease-related modules, GO and KEGG functional enrichment analysis was performed on the genes from the 7 modules. We observed that these 7 modules were enriched in GO terms and KEGG pathways (Figure $4 A$ ). Specifically, the brown and orangered 4 modules were mainly enriched in cell components; darkgreen was mainly enriched in biological processes and KEGG pathway; grey60 was mainly enriched in cell components and biological processes; lightgreen module was mainly enriched in biological processes and molecular functions; and mediumpurple3 


\begin{tabular}{|c|c|c|c|c|c|c|c|}
\hline \multicolumn{7}{|l|}{$A$} & \multirow{5}{*}{ 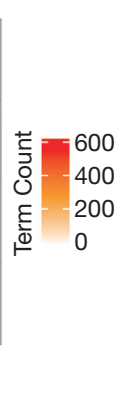 } \\
\hline Biological_Process & 0.00 & 532.00 & 82.00 & 15.00 & 48.00 & 0.00 & \\
\hline Cellular_Component & 3.00 & 8.00 & 36.00 & 2.00 & 0.00 & 4.00 & \\
\hline Molecular_Function & 1.00 & 12.00 & 6.00 & 19.00 & 4.00 & 0.00 & \\
\hline Pathway_KEGG & 0.00 & 60.00 & 9.00 & 1.00 & 0.00 & 0.00 & \\
\hline
\end{tabular}
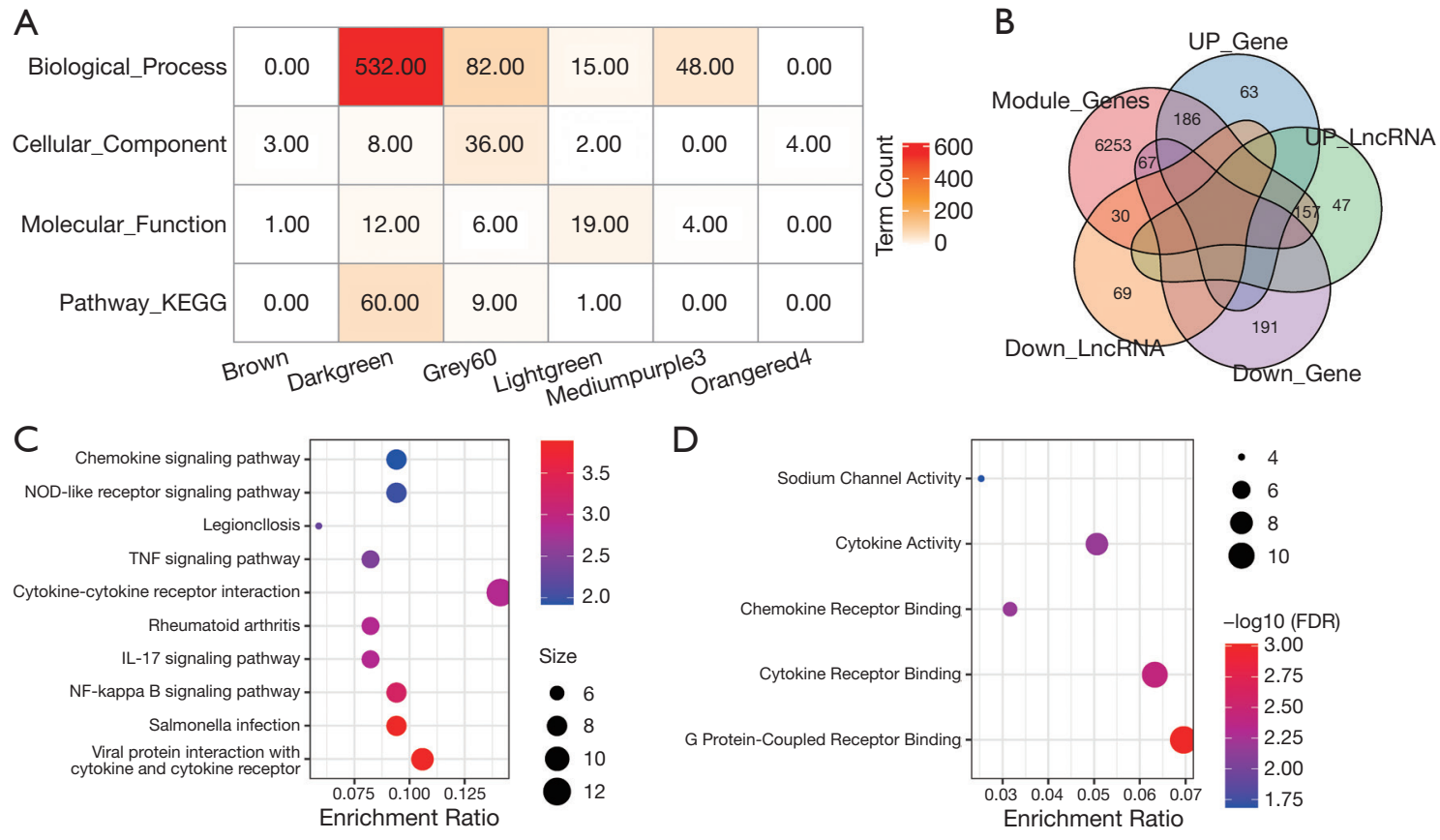

Figure 4 Functional enrichment analysis of disease-related modules. (A) GO term and KEGG pathway statistics of the 7 modules; (B) Wayne diagram of the intersection between genes and lncRNAs and DEGs and DElncRNAs in the co-expression module; (C) KEGG pathway enrichment results of differentially co-expressed genes. (D) GO term enrichment results of differentially co-expressed genes. Different colors indicate the significance of enrichment, and dot size indicates the number of genes enriched. GO, Gene Ontology; KEGG, Kyoto Encyclopedia of Genes and Genomes; DEG, differentially expressed gene; DElncRNA, differentially expressed long non-coding RNA.

was mainly enriched in biological processes. These results indicated that different modules may be involved in different biological pathways. Similarly, we statistically analyzed the intersection of genes and lncRNAs in these 7 modules with differential genes and lncRNAs (Figure 4B). It was observed that there were 157 up-regulated lncRNAs and 186 upregulated genes, 30 down-regulated lncRNAs and 67 downregulated genes in the co-expression module. Further enrichment analysis of these DEGs in KEGG pathways and GO terms showed that these genes were mainly enriched in cytokine and cytokine receptor, salmonella infection, NF-kappa B signaling pathway, interleukin-17 (IL-17) signaling pathway, rheumatoid arthritis, cytokinecytokine receptor interaction, and tumor necrosis factor (TNF) signaling pathway, legionellosis, NOD-like receptor signaling pathway, and other inflammatory and immunerelated pathways (Figure 4C). In addition, they also enriched in $\mathrm{G}$ protein-coupled receptor binding, cytokine receptor binding, chemokine receptor binding, cytokine activity, and other immune-related molecular functions (Figure 4D). Inflammation also plays an important role in the occurrence of CAD, and both the innate immune system and adaptive immune system have critical functions in initiation and progression of atherosclerosis. Therefore, these differentially co-expressed genes may be the key genes in CAD.

\section{Identification of LRM-CAD and its role in CAD}

We employed a new computational method for identifying LRM-CAD of CAD through integrating DEGs/ DElncRNA matched expression profiles from diseaseassociated co-expression modules into a gene expression dataset based on regulatory interactions among mRNAs, lncRNAs, and miRNAs. Firstly, we compared the genomic distances between the DEGs and DElncRNAs in the same co-expression module, and mapped the genes and lncRNAs onto the genome. When lncRNAs were upstream of the genes, they were considered potential cisregulatory pairs, otherwise they were regarded as transregulatory pairs. In this way, 235 cis-regulatory pairs and 25,629 trans-regulatory pairs were obtained. We further 

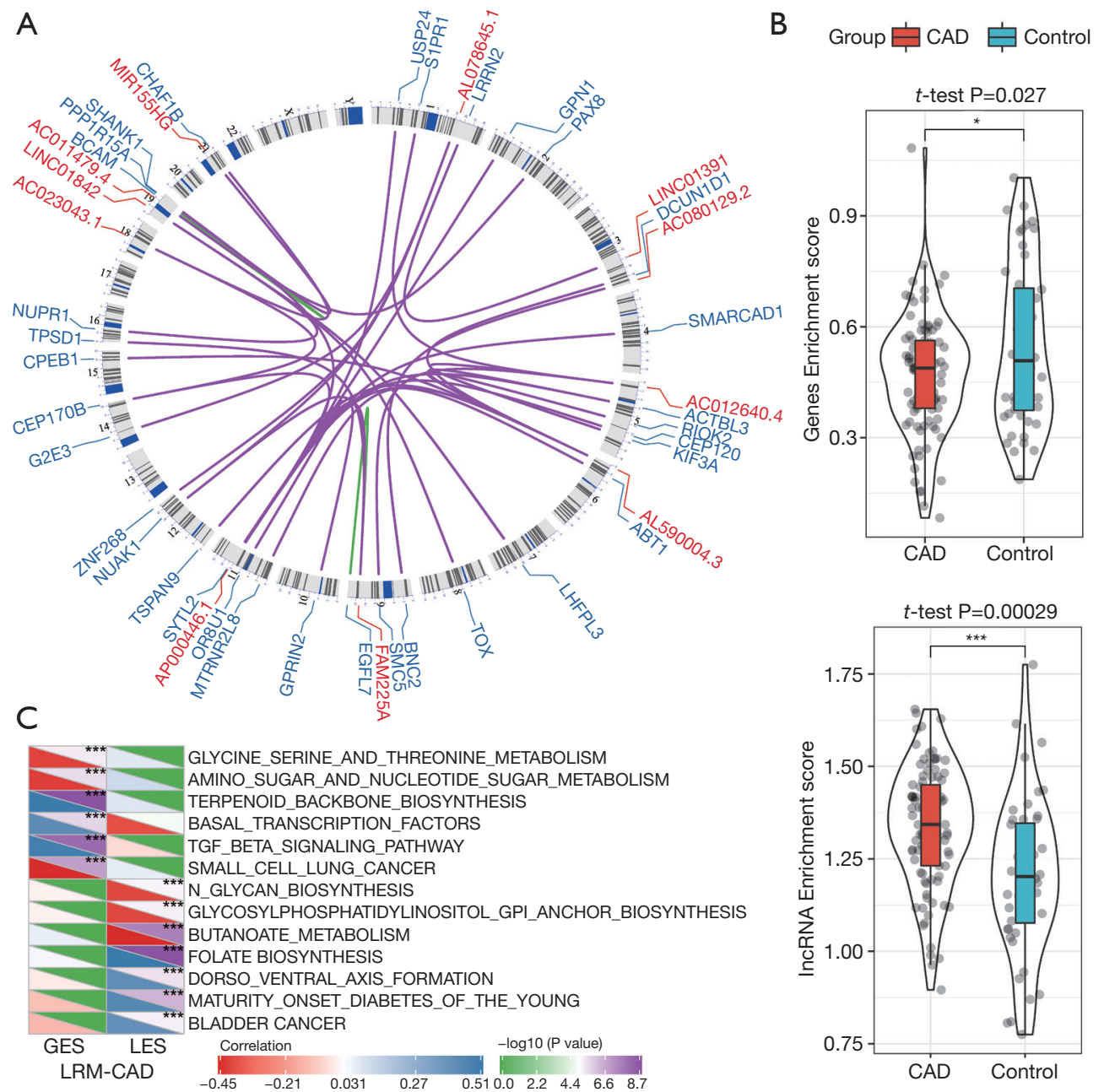

Figure 5 Genomic distribution and potential function of LRM-CAD. (A) Genomic distribution of genes and lncRNAs in LRM-CAD. (B) The distribution difference of gene and lncRNA enrichment scores in single sample of LRM-CAD in CAD samples and control samples. (C) KEGG pathway significantly associated with LRM-CAD-GES and LRM-CAD-LES. KEGG, Kyoto Encyclopedia of Genes and Genomes; LRM-CAD, dysregulated lncRNA-mRNA; LRM-CAD-GES, LRM-CAD-genes enrichment scores; LRM-CAD-LES, LRM-CADlncRNA enrichment scores. ${ }^{*} \mathrm{P}<0.05,{ }^{* * *} \mathrm{P}<0.001$.

analyzed the miRNAs of targeted genes and lncRNAs in each regulation pair, counted the number of miRNAs shared by genes and IncRNAs, and used hypergeometry to evaluate the significance of miRNAs shared by genes and lncRNAs in each regulation pair. Finally, 36 regulatory pairs of significantly shared miRNAs as LRM-CAD (Figure $5 A$ ), including $11 \mathrm{lncRNAs}$ and 33 genes, were acquired, and on the whole, trans-regulation was the mainstay. We verified the role of these LRM-CADs in CAD from multiple perspectives. We first calculated the enrichment score of 33 genes (LRM-CAD-GES) and 11 lncRNA enrichment scores (LRM-CAD- LES) (Figure 5B), which revealed significant differences between them in CAD and healthy control samples, especially, lncRNA showed a significant difference in CAD. Furthermore, the singlesample gene set enrichment analysis method was used to calculate the enrichment score of each KEGG pathway, and the correlation between LRM-CAD-GES/LRM-CADLES and the enrichment score of each KEGG pathway was evaluated. Here, a total of 13 KEGG pathways were obtained (FDR $<0.05$, Figure 5 C). Specifically, 6 pathways were found to be significantly related to LRM-CAD-GES, including transforming growth factor (TGF)-beta signaling pathway and pathways related to amino acid metabolism, 
and 7 pathways, including maturity-onset diabetes of the young and multiple pathways related to energy metabolism, were significantly associated with LRM-CAD-LES. These data suggested that the occurrence of CAD may be associated with some metabolic disorders.

\section{LRM-CAD as a diagnostic biomarker for CAD}

Considering the differences between LRM-CADGES and LRM-CAD-LES in CAD, we evaluated the CAD classification performance of each LRM-CAD and single LRM-CAD gene and single LRM-CAD lncRNA, respectively. For each LRM-CAD lncRNA, their expression level showed a high diagnostic performance for CAD classification ROC (Figure 6A). Similarly, for each LRM-CAD gene, they also showed a strong diagnostic performance (Figure 6B). Furthermore, linear discriminant analysis was performed to classify and predict each LRMCAD and ROC analysis. It was observed that compared with a single lncRNA or gene, these LRM-CAD had higher classification performance (Figure $6 C$ ) with an average AUC of 0.958 , suggesting that these LRM-CAD may be a potential diagnostic marker of CAD.

\section{Identification of potential drugs targeting LRM-CAD}

To further identify the therapeutic drugs for LRMCAD, we obtained 5,490 drug-protein interaction data from the Drugbank database, and constructed a drugprotein interaction network using the protein interaction information in the STRING database. The genes in LRMCAD were mapped to the network to calculate the closeness of the drug to LRM-CAD, and a random network was constructed using stochastic simulation as a background. The proximity distribution of the final drug to LRM-CAD was smaller than that of the random background drug to LRM-CAD (Figure 7A). We selected drugs with a global FDR $<0.05$, which yielded 3 drugs (Table 1). These 3 drugs directly interact with the S1PR1 gene in LRM-CAD. We used molecular docking methods to verify the binding ability of S1PR1 with DB09105, DB12371, and DB12612. Considering that DB09105 was a protein molecule, we used the 2 small molecule compounds DB12371 and DB12612 for molecular docking. Both DB12371 and DB12612 showed high docking scores, the docking score of compound DB12612 and S1PR1 reached $-9.4 \mathrm{kcal} / \mathrm{mol}$ (Figure $7 B$ ), and DB12371, which had a docking score of $-11.1 \mathrm{kcal} / \mathrm{mol}$ (Figure $7 C$ ). To a certain extent, these data indicated that these 2 compounds and S1PR1 could be relatively tightly combined and had a potential biological activity.

\section{Verification of diagnostic performance of S1PR1- AC012640.4}

The gene S1PR1 may be the direct target of DB09105, DB12371, and DB12612. Here, external data sets GSE20681 and GSE64566 were used as validation sets to evaluate the diagnostic performance of $S 1 P R 1$ and $A C 012640.4$. The expression profiles of $S 1 P R 1$ and $A C 012640.4$ were obtained from the GSE20681 data set. The expression level of S1PR1 was used to predict CAD, and the ROC analysis revealed an AUC of 0.773 (Figure $8 \mathrm{~A}$ ). The expression level of $A C 012640.4$ was used to predict CAD, and ROC analysis showed that the AUC was 0.755 (Figure $8 B$ ). Combining predictive CAD with S1PR1AC012640.4, ROC analysis showed an AUC of 0.79 (Figure $8 C$ ). In addition, only S1PR1 was detected in the GSE64566 dataset, thus, we also analyzed the diagnostic performance of S1PR1 in the GSE64566 dataset. The ROC showed that S1PR1 expression still had a strong diagnostic performance (AUC $=0.754$, Figure $8 D$ ). These results indicated that S1PR1, AC012640.4 and S1PR1-AC012640.4 could effectively distinguish CAD from control, and that these genes and lncRNAs may be reliable biomarkers for CAD specific diagnosis.

\section{Discussion}

In this study, we obtained a relatively large sample (93 CAD patients and 48 healthy controls) of whole-transcriptome lncRNA and mRNA expression data by high-throughput microarray screening. To determine the function of DElncRNAs, 7 CAD-related modules were identified by WGCNA and analyzed for GO and KEGG pathways. It was found that some differentially expressed mRNAs were involved in inflammatory cytokine and immune cell secretion pathways. Proinflammatory cytokines and chemokines have been reported to be involved in all stages of atherosclerotic lesion development. In addition, 36 regulatory pairs of significantly shared by miRNAs were considered as LRM-CAD, including 11 lncRNAs and 33 genes. The ROC analysis demonstrated that LRMCAD had a higher classification performance. We screened 3 potential therapeutic drugs DB09105, DB12371 and DB12612 by drug-target gene interaction network, and 
A

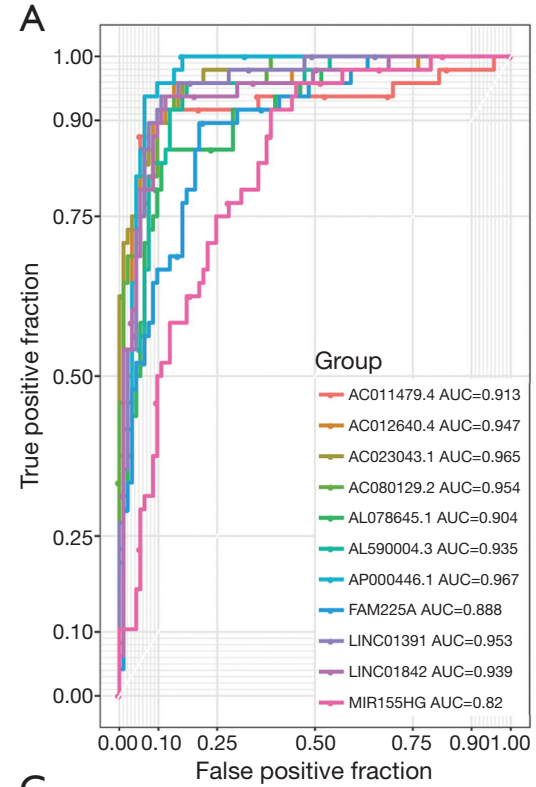

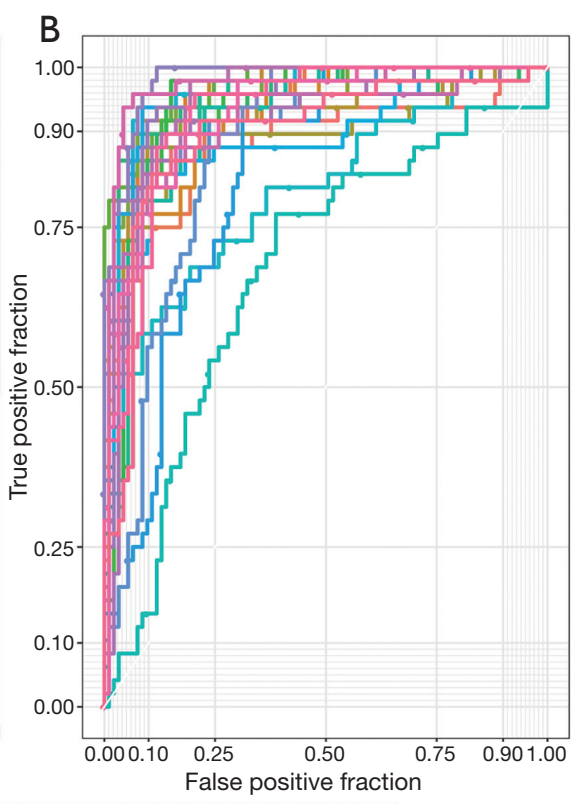

Group

- $\mathrm{ABT} 1 \mathrm{AUC}=0.888 \quad$ NUAK1 $\mathrm{AUC}=0.802$

- ACTBL2 AUC $=0.921 \quad$ NUPR1 AUC $=0.961$

- BCAM AUC $=0.949-$ OR8U1 AUC $=0.88$

-BNC2 AUC $=0.907-$ PAX8 AUC $=0.963$

- CEP120 AUC $=0.957-$ PPP1R15A AUC $=0.817$

- CEP170B AUC $=0.917$ - RIOK2 AUC $=0.858$

- CHAF1B AUC $=0.893 \quad-\mathrm{S} 1 \mathrm{PR} 1 \mathrm{AUC}=0.94$

- CPEB1 AUC $=0.942-$ SHANK1 AUC $=0.982$

-DCUN1D1 AUC $=0.96-$ SMARCAD1 AUC $=0.935$

- EGFL7 AUC $=0.97-\mathrm{SMC5}$ AUC $=0.906$

- G2E3 AUC $=0.923 \quad$ SYTL2 AUC $=0.953$

-GPN1 AUC $=0.944 \quad-$ TOX AUC $=0.913$

- GPRIN2 AUC $=0.915-$ TPSD1 AUC $=0.967$

-KIF3A AUC $=0.955 \quad-$ TSPAN9 AUC $=0.926$

- LHFPL3 AUC $=0.921-$ USP24 AUC $=0.928$

- LRRN2 AUC $=0.928 \quad$ ZNF268 AUC $=0.894$

-MTRNR2L8 AUC $=0.68$
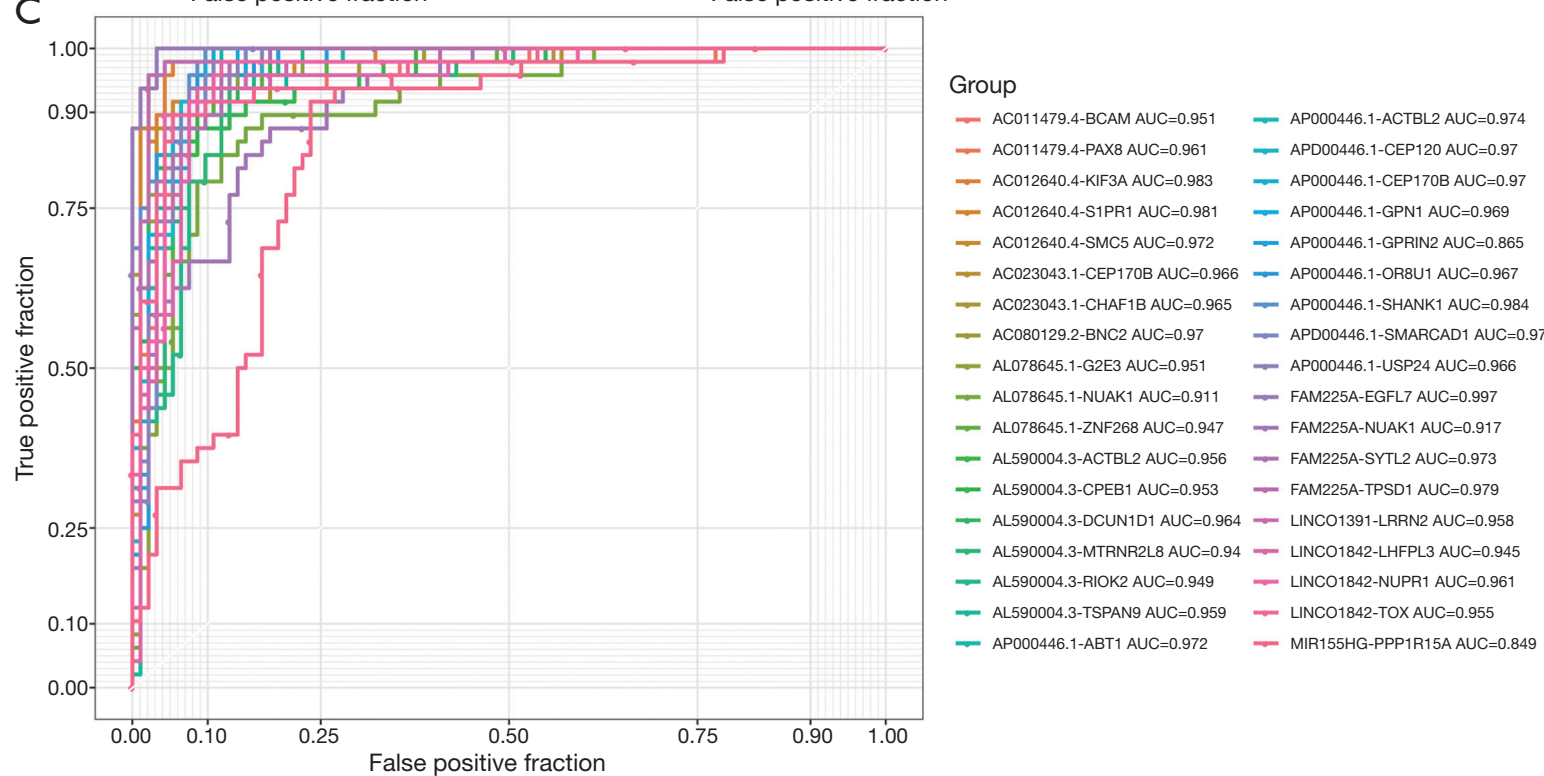

Figure 6 ROC analysis of LRM-CAD as diagnostic markers. (A) ROC analysis of 11 lncRNAs in LRM-CAD. (B) ROC analysis of 33 genes in LRM-CAD. (C) ROC analysis of 36 LRM-CAD. ROC, receiver operating characteristic; LRM-CAD, dysregulated lncRNA-mRNA.

there was a direct interaction with the S1PR1 gene in LRM-CAD. External data sets verified that S1PR1-lncRNA AC012640.4 could effectively distinguish CAD from control.

As a leading cause of death worldwide, and early prevention of CAD can reduce morbidity and mortality. Therefore, this study was designed to identify new biomarkers to improve the prediction and treatment of CAD. Many studies have confirmed that circulating miRNAs can be used as disease markers in blood of cardiovascular diseases such as acute myocardial infarction (AMI) and heart failure (34). Exosomal microRNAs have been reported to play an important role in the progress of CAD. For example, Exosomal microRNA-25-3p inhibits coronary vascular endothelial cell inflammation (35), Exosomal microRNA-21, microRNA-126, and PTEN are novel biomarkers for diagnosis of acute coronary syndrome (36). Similar to miRNAs and Exosomal microRNAs, lncRNAs can also be 

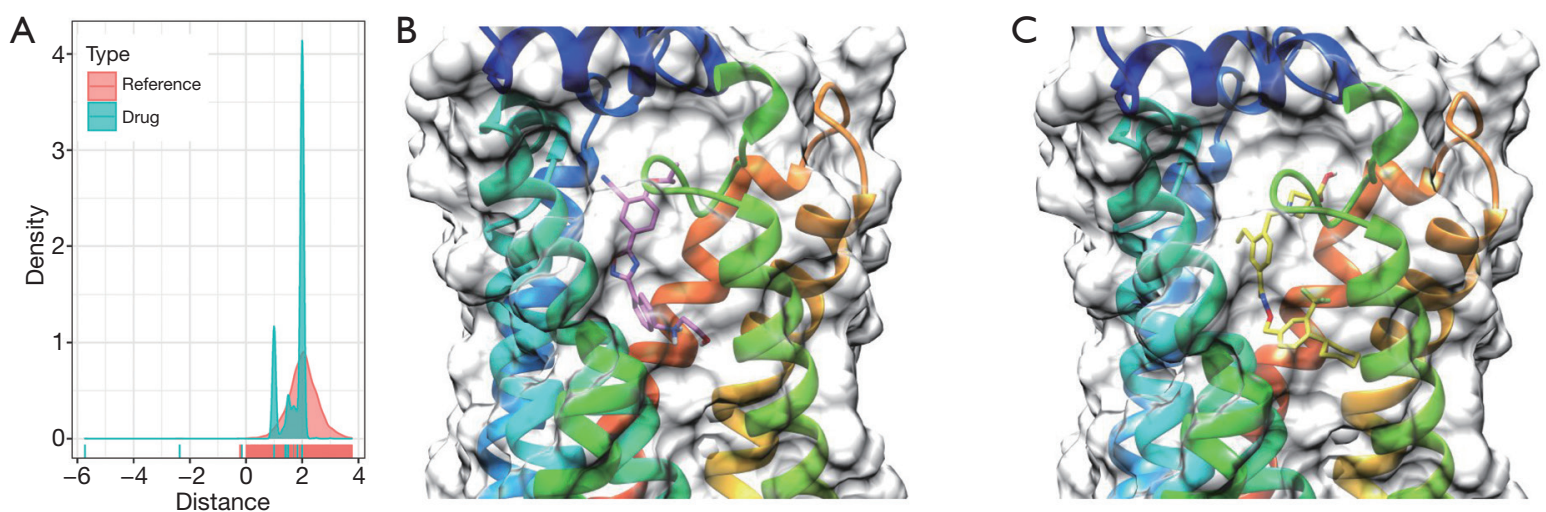

Figure 7 Potential drug analysis of LRM-CAD. (A) Proximity distribution between drugs and LRM-CAD. (B) Interaction between DB12612 and S1PR1. (C) The interaction between DB12371 and S1PR1; compound DB12371 is shown as yellow, DB12612 as orchid, and the protein surface as white. LRM-CAD, dysregulated lncRNA-mRNA.

Table 1 Informations of 3 drugs

\begin{tabular}{lccccc}
\hline Drug_id & Distances & P value & FDR & global_pvalue & global_FDR \\
\hline DB09105 & -5.7301 & $2.53 \mathrm{E}-41$ & $1.39 \mathrm{E}-37$ & $3.52 \mathrm{E}-51$ & $9.93 \mathrm{E}-47$ \\
DB12371 & -2.36505 & $5.01 \mathrm{E}-19$ & $2.75 \mathrm{E}-15$ & $5.43 \mathrm{E}-14$ \\
DB12612 & -2.36505 & $5.01 \mathrm{E}-19$ & $2.75 \mathrm{E}-15$ & $9.89 \mathrm{E}-18$ & $5.43 \mathrm{E}-14$ \\
\hline
\end{tabular}

FDR, false discovery rate.

detected in blood and could also serve as biomarkers (37). In a study by Vausort et al., 5 lncRNAs, including AHIF, ANRIL, KCNQ1QT1, MIAT, and MALAT1, were detected in peripheral blood mononuclear cell (PBMC) of 414 AMI patients and 86 control patients (38). The lncRNAs KCNQ1QT1, AHIF, and MALAT1 in AMI patients were found to be higher than those in the control group, and ANRIL was lower than control group. Meanwhile, ANRIL, KCNQ1OT1, MIAT, and MALAT1 were lower in patients with ST segment elevation MI than in those without. Finally, ANRIL and KCNQ1QT1 were considered capable of predicting left ventricular dysfunction after AMI. Yang et al. detected the expression of lncRNA in 20 patients with CAD and 20 controls (39). Serum lncRNA AC100865.1, monocyte LncPPARd (40), and OTTHUMT00000387022 were detected as markers for the diagnosis of CAD. In this study, bioinformatics study showed that S1PR1-AC012640.4 could effectively distinguish CAD from healthy groups and serve as a biomarker for $\mathrm{CAD}$ specific diagnosis.

Overexpression of fibroblast-specific S1PR1 in mouse hearts has been shown to increase cardiac tissue hypertrophy and fibrosis, accompanied by up-regulation of signal transduction and transcriptional activator 3 (STAT3) signal transduction and interleukin-6 (IL-6) production (41). The drug-protein interaction network showed that both DB12371 and DB12612 could bind to S1PR1 well, suggesting that DB12371 and DB12612 might be potential drugs targeting S1PR1.

Although we analyzed and validated the abnormal expression and functional role of genes in CAD through bioinformatics with multiple data coalitions, some limitations of this study should be noted. Firstly, the sample lacked some clinical follow-up information, therefore we did not consider factors such as the presence of patient comorbidities in distinguishing the biomarkers. Secondly, the results were obtained only by bioinformatics analysis, which was insufficient, and experimental validation is needed to confirm these results. Therefore, further genetic and experimental studies with larger sample sizes and experimental validation are required.

\section{Conclusions}

In this study, we systematically analyzed gene expression 

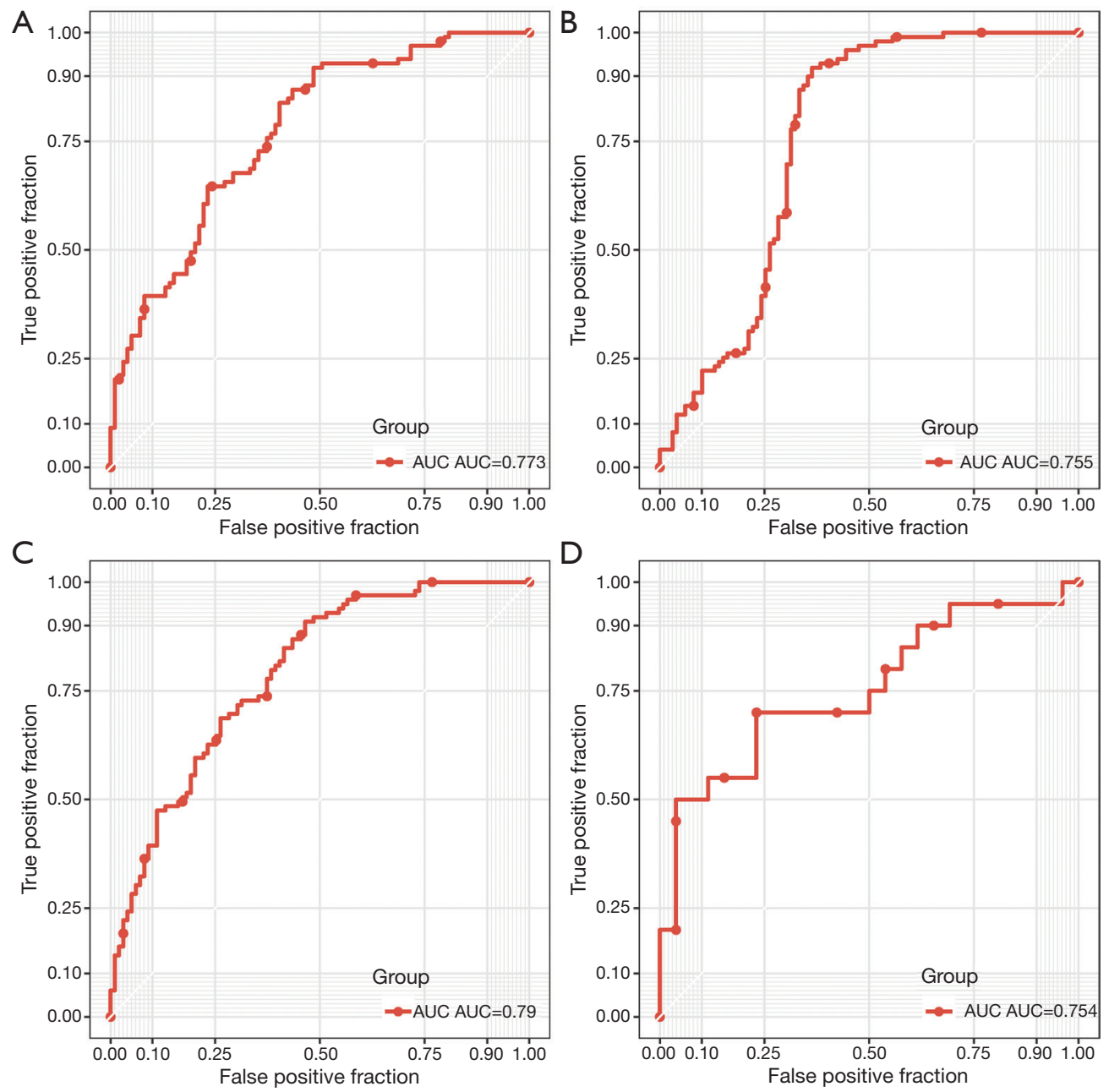

Figure 8 Diagnostic performance of S1PR1-AC012640.4 in an external validation set. (A) ROC analysis of S1PR1 gene in GSE20681 data set. (B) ROC analysis of lncRNA AC012640.4 in GSE20681 dataset. (C) ROC analysis of S1PR1-AC012640.4 in GSE20681 data set; (D) ROC analysis of S1PR1 gene in GSE64566 data set. ROC, receiver operating characteristic.

patterns in CAD and conducted a large-scale genome-wide study on RNA expression profiles to identify gene modules closely related to CAD. Through disease association network mining, we found 3 potential drugs in CAD, providing targets and reference for clinicians and biological experimentalists.

\section{Acknowledgments}

Funding: None.

\section{Footnote}

Reporting Checklist: The authors have completed the
TRIPOD reporting checklist. Available at https://dx.doi. org/10.21037/atm-21-3276

Conflicts of Interest: All authors have completed the ICMJE uniform disclosure form (available at https://dx.doi. org/10.21037/atm-21-3276). The authors have no conflicts of interest to declare.

Ethical Statement: The authors are accountable for all aspects of the work in ensuring that questions related to the accuracy or integrity of any part of the work are appropriately investigated and resolved. The study was conducted in accordance with the Declaration of Helsinki (as revised in 2013). 
Open Access Statement: This is an Open Access article distributed in accordance with the Creative Commons Attribution-NonCommercial-NoDerivs 4.0 International License (CC BY-NC-ND 4.0), which permits the noncommercial replication and distribution of the article with the strict proviso that no changes or edits are made and the original work is properly cited (including links to both the formal publication through the relevant DOI and the license). See: https://creativecommons.org/licenses/by-nc-nd/4.0/.

\section{References}

1. Benjamin EJ, Virani SS, Callaway CW, et al. Heart Disease and Stroke Statistics-2018 Update: A Report From the American Heart Association. Circulation 2018;137:e67-e492.

2. Chiu MH, Heydari B, Batulan Z, et al. Coronary artery disease in post-menopausal women: are there appropriate means of assessment? Clin Sci (Lond) 2018;132:1937-52 .

3. Madhavan MV, Gersh BJ, Alexander KP, et al. Coronary Artery Disease in Patients $\geq 80$ Years of Age. J Am Coll Cardiol 2018;71:2015-40.

4. Zhang HW, Jin JL, Cao YX, et al. Heart-type fatty acid binding protein predicts cardiovascular events in patients with stable coronary artery disease: a prospective cohort study. Ann Transl Med 2020;8:1349.

5. Wykrzykowska JJ, Garcia-Garcia HM, Goedhart D, et al. Differential protein biomarker expression and their timecourse in patients with a spectrum of stable and unstable coronary syndromes in the Integrated Biomarker and Imaging Study-1 (IBIS-1). Int J Cardiol 2011;149:10-6.

6. Batista PJ, Chang HY. Long noncoding RNAs: cellular address codes in development and disease. Cell 2013;152:1298-307.

7. Yan HX, Du J, Fu J, et al. Microarray-based differential expression profiling of long noncoding RNAs and messenger RNAs in formalin-fixed paraffin-embedded human papillary thyroid carcinoma samples. Transl Cancer Res 2019;8:439-51.

8. Schunkert H, König IR, Kathiresan S, et al. Large-scale association analysis identifies 13 new susceptibility loci for coronary artery disease. Nat Genet 2011;43:333-8.

9. Amaral PP, Dinger ME, Mercer TR, et al. The eukaryotic genome as an RNA machine. Science 2008;319:1787-9.

10. Shukla GC, Singh J, Barik S. MicroRNAs: Processing, Maturation, Target Recognition and Regulatory Functions. Mol Cell Pharmacol 2011;3:83-92.

11. Singleton PA, Dudek SM, Chiang ET, et al. Regulation of sphingosine 1-phosphate-induced endothelial cytoskeletal rearrangement and barrier enhancement by S1P1 receptor, PI3 kinase, Tiam1/Rac1, and alpha-actinin. Faseb J 2005;19:1646-56.

12. Karliner JS. Sphingosine kinase and sphingosine 1-phosphate in the heart: a decade of progress. Biochim Biophys Acta 2013;1831:203-12.

13. Park SJ, Kim JM, Kim J, et al. Molecular mechanisms of biogenesis of apoptotic exosome-like vesicles and their roles as damage-associated molecular patterns. Proc Natl Acad Sci U S A 2018;115:E11721-30.

14. Zervantonakis IK, Iavarone C, Chen HY, et al. Systems analysis of apoptotic priming in ovarian cancer identifies vulnerabilities and predictors of drug response. Nat Commun 2017;8:365.

15. Li L, Wang L, Li H, et al. Characterization of LncRNA expression profile and identification of novel LncRNA biomarkers to diagnose coronary artery disease. Atherosclerosis 2018;275:359-67.

16. Elashoff MR, Wingrove JA, Beineke P, et al. Development of a blood-based gene expression algorithm for assessment of obstructive coronary artery disease in non-diabetic patients. BMC Med Genomics 2011;4:26.

17. Vacca M, Di Eusanio M, Cariello M, et al. Integrative miRNA and whole-genome analyses of epicardial adipose tissue in patients with coronary atherosclerosis. Cardiovasc Res 2016;109:228-39.

18. Birney E, Stamatoyannopoulos JA, Dutta A, et al. Identification and analysis of functional elements in $1 \%$ of the human genome by the ENCODE pilot project. Nature 2007;447:799-816.

19. Ritchie ME, Phipson B, Wu D, et al. limma powers differential expression analyses for RNA-sequencing and microarray studies. Nucleic Acids Res 2015;43:e47.

20. Langfelder P, Horvath S. WGCNA: an R package for weighted correlation network analysis. BMC Bioinformatics 2008;9:559.

21. Yu G, Wang LG, Han Y, et al. clusterProfiler: an R package for comparing biological themes among gene clusters. OMICS 2012;16:284-7.

22. Hanzelmann S, Castelo R, Guinney J. GSVA: gene set variation analysis for microarray and RNA-seq data. BMC Bioinformatics 2013;14:7.

23. Miranda KC, Huynh T, Tay Y, et al. A pattern-based method for the identification of MicroRNA binding sites and their corresponding heteroduplexes. Cell 2006;126:1203-17.

24. Huang HY, Lin YC, Li J, et al. miRTarBase 2020: 
updates to the experimentally validated microRNAtarget interaction database. Nucleic Acids Res 2020;48:D148-D54.

25. Lewis BP, Burge CB, Bartel DP. Conserved seed pairing, often flanked by adenosines, indicates that thousands of human genes are microRNA targets. Cell 2005;120:15-20.

26. Li JH, Liu S, Zhou H, et al. starBase v2.0: decoding miRNA-ceRNA, miRNA-ncRNA and protein-RNA interaction networks from large-scale CLIP-Seq data. Nucleic Acids Res 2014;42:D92-7.

27. Jeggari A, Marks DS, Larsson E. miRcode: a map of putative microRNA target sites in the long non-coding transcriptome. Bioinformatics 2012;28:2062-3.

28. Salmena L, Poliseno L, Tay Y, et al. A ceRNA hypothesis: the Rosetta Stone of a hidden RNA language? Cell 2011;146:353-8.

29. Tay Y, Rinn J, Pandolfi PP. The multilayered complexity of ceRNA crosstalk and competition. Nature 2014;505:344-52.

30. Hu Y, Yan C, Hsu CH, et al. OmicCircos: A Simpleto-Use R Package for the Circular Visualization of Multidimensional Omics Data. Cancer Inform 2014;13:13-20.

31. Wishart DS, Feunang YD, Guo AC, et al. DrugBank 5.0: a major update to the DrugBank database for 2018. Nucleic Acids Res 2018;46:D1074-82.

32. Peng Y, Yuan M, Xin J, et al. Screening novel drug candidates for Alzheimer's disease by an integrated network and transcriptome analysis. Bioinformatics 2020;36:4626-32.

33. Szklarczyk D, Gable AL, Lyon D, et al. STRING v11: protein-protein association networks with increased coverage, supporting functional discovery in genome-

Cite this article as: Chen Z, Zhou D, Zhang X, Wu Q, Wu G. Diagnostic biomarkers and potential drug targets for coronary artery disease as revealed by systematic analysis of lncRNA characteristics. Ann Transl Med 2021;9(15):1243. doi: 10.21037/atm-21-3276 wide experimental datasets. Nucleic Acids Res 2019;47:D607-13.

34. Shin VY, Chu KM. MiRNA as potential biomarkers and therapeutic targets for gastric cancer. World J Gastroenterol 2014;20:10432-9.

35. Yao Y, Sun W, Sun Q, et al. Platelet-Derived Exosomal MicroRNA-25-3p Inhibits Coronary Vascular Endothelial Cell Inflammation Through Adam 10 via the NF- B Signaling Pathway in ApoE(-/-) Mice. Front Immunol 2019;10:2205.

36. Ling H, Guo Z, Shi Y, et al. Serum Exosomal MicroRNA-21, MicroRNA-126, and PTEN Are Novel Biomarkers for Diagnosis of Acute Coronary Syndrome. Front Physiol 2020;11:654.

37. Reis EM, Verjovski-Almeida S. Perspectives of Long Non-Coding RNAs in Cancer Diagnostics. Front Genet 2012;3:32.

38. Vausort M, Wagner DR, Devaux Y. Long noncoding RNAs in patients with acute myocardial infarction. Circ Res 2014;115:668-77.

39. Yang Y, Cai Y, Wu G, et al. Plasma long non-coding RNA, CoroMarker, a novel biomarker for diagnosis of coronary artery disease. Clin Sci (Lond) 2015;129:675-85.

40. Cai Y, Yang Y, Chen X, et al. Circulating "LncPPAR " From Monocytes as a Novel Biomarker for Coronary Artery Diseases. Medicine (Baltimore) 2016;95:e2360.

41. Ohkura SI, Usui S, Takashima SI, et al. Augmented sphingosine 1 phosphate receptor-1 signaling in cardiac fibroblasts induces cardiac hypertrophy and fibrosis through angiotensin II and interleukin-6. PLoS One 2017;12:e0182329.

(English Language Editor: J. Jones) 